\title{
Die Homöopathie im Spannungsfeld zwischen aristotelischer und moderner Wissenschaft
}

\author{
Josef M. Schmidt \\ Institut für Geschichte der Medizin, Ludwig-Maximilians-Universität München, Deutschland
}

$V$ or über 200 Jahren, im Jahre 1807 , prägte SAMUEL HaHnEmann den Begriff „homöopathisch“ für die von ihm entdeckte neue Heilmethode. Das Prinzip, auf dem sie beruhte, nämlich Krankheitszustände mit solchen Mitteln zu behandeln, die in Arzneimittelprüfungen an Gesunden ähnliche Krankheitszustände hervorrufen, hatte er schon 1796 publiziert (Similia similibus) [1]. Doch noch 1805 , als er seine neue Lehre bereits lehrbuchartig in einer Monographie darstellte, bezeichnete er sie immer noch lediglich als „Heilkunde der Erfahrung“ [2]. Erst 1807 nannte er die Wirkung bestimmter heilender Arzneimittel „homöopathisch“ und definierte: „Homöopathisch ist, was ein hómoion páthos, ein ähnliches Leiden, zu erzeugen Tendenz hat“ [3].

Durch diese neue Wortschöpfung erhielt die Homöopathie ihren Namen, wodurch sie erst so etwas wie eine eigene Entität (Wesenheit) wurde, die als solche nun ihren Weg durch die Geschichte und um die ganzen Welt antreten konnte. Was die seitdem erreichte raum-zeitliche Ausbreitung und Institutionalisierung betrifft, kann die Homöopathie heute auf eine beeindruckende Karriere zurückblicken. Bei genauerem Hinsehen erweist sich jedoch das Subjekt dieser Geschichte als nicht besonders homogen. Seit jeher hatte es hinsichtlich der eigenen Lehre interne inhaltliche Differenzen gegeben, die wiederholt auch Anlass für Spaltungen und Schulbildungen gaben [4]. Mittlerweile wird der Markenname „Homöopathie“ von einer Vielzahl unterschiedlicher Strömungen für sich in Anspruch genommen, so dass es nicht leicht ist, eine einheitliche, für alle diese Ansätze gültige Definition des Begriffs zu erhalten. Einer der zentralsten Punkte der aktuellen Diskussion über das We-

Die Frage nach der Wissenschaftlichkeit der Homöopathie wird heute meist ausschliesslich vor dem Hintergrund des neuzeitlichen Begriffs von Naturwissenschaft diskutiert. Dabei wird übersehen, dass die Homöopathie - wissenschaftsgeschichtlich betrachtet - mehrere Wurzeln hat, die sich im Wesentlichen auf zwei wirkmächtige Wissenschaftstraditionen zurückführen lassen Zum einen Prinzipien und Begriffe des Aristotelismus, die 2000 Jahre die abendländische Wissenschaftsgeschichte dominierten, zum anderen das moderne Konzept von Naturwissenschaft das erst seit weniger als 200 Jahren die Medizingeschichte bestimmt. Umfasste Aristoteles' "Wissenschaft vom Lebendigen" noch ontologische und teleologische Dimensionen zum Zwecke eines einheitlichen Naturverständnisses, so verengte sich das neuzeitliche naturwissenschaftliche Interesse auf funktionale und kausale Erklärungen der Phänomene im Hinblick auf Naturbeherrschung. Nicht nur zur Verhinderung weiterer ökologischer Katastrophen, sondern auch zur Rückgewinnung verlorengegangener Dimensionen unseres Lebens sollte die Einseitigkeit und Theorielastigkeit unseres modernen naturwissenschaftlichen Weltbildes durch ein Gegengewicht lebensweltlich-praktischer aristotelischer Kategorien ausbalanciert werden. Auf diese Weise liesse sich auch die Frage der Wissenschaftlichkeit der Homöopathie in einem breiteren, eher aristotelischen Sinne neu stellen und diskutieren.

Schlüsselwörter: Homöopathie, Wissenschaft, moderne Naturwissenschaft, Aristoteles, Hahnemann

\begin{abstract}
Homeopathy in the Tension Field between Aristotelic and Modern Sciences
Today, the question whether homeopathy is scientific or not is often discussed with reference to modern natural sciences. Doing so, one fails to realise that homeopathy - from a historical perspective - originates from two distinct roots both of which have evolved from an influentia scientific tradition: principles and notions of Aristotelism on the one hand, and modern concepts of natural sciences on the other. While Aristotle's 'science of the living' comprised ontologic and teleologic dimensions for a global comprehension of nature, modern natural sciences are only interested in functional and causal explanations of phenomena for the purpose of dominating nature. Homeopathy was founded at the point of intersection of these traditions. In times of increasing awareness of the side effects of the natural-science approach, such as ecological catastrophes or a general loss of perception of various dimensions of our lives, the modern natural-science view of life should be counterbalanced by lifeworld-related Aristotelic categories. Accordingly, the scientific character of homeopathy might be conceived in an Aristotelian sense rather than exclusively in terms of modern natural sciences.
\end{abstract}

Key Words: Homeopathy, science, modern natural science, Aristotle, Hahnemann

sen der Homöopathie - die im Einzelnen im Internet nachzulesen ist [5] ist die alte grundsätzliche Frage, die Homöopathen und ihre Kritiker seit jeher beschäftigt, ob die Homöopathie eine Wissenschaft sei, und wenn ja, was für eine Art von Wissenschaft.

Das Spektrum der Antworten reicht momentan von der These, die Homöopathie gehöre in die hermetisch-esoterische Tradition der Alchimie bzw. des Schamanismus und wäre gut beraten, dies offen zuzugeben und sich gerade nicht als naturwissenschaftliche Medi- zin zu definieren [6], bis zu dem Anspruch, Homöopathie sei die einzige Form von Medizin, die dem neuzeitlichen Ideal von Wissenschaft als eines apriorisch-sicheren, mathematischen Wissens entspreche [7]. Zwischen diesen Extrem-Positionen finden sich auch Meinungen wie: Eine künftige evidence-based homeopathy könnte den Durchbruch in der Anerkennung als Wissenschaft bringen [8]; oder aber Standpunkte wie: Als praktische Heilungs-Wissenschaft habe sich die Homöopathie ausschliesslich am indivi- 
duellen Einzelfall in der Praxis zu bewähren und sollte auf kontrollierte klinische Studien verzichten [9]; usw.

Auseinandersetzungen wie diese, wo es um das Verhältnis von Homöopathie und Wissenschaft geht, sind oft gerade deshalb so langlebig und schwer aufzulösen, weil die Begriffe, um die es geht, bereits eine Entwicklung von mehreren Jahrhunderten bzw. mehreren Jahrtausenden hinter sich haben und dementsprechend viele Traditionen und Bedeutungen umfassen. So mag es sinnvoll sein, erst einmal einen Schritt zurückzutreten und sich zu vergegenwärtigen, was das eigentlich jeweils heisst bzw. sein soll: Wissenschaft und Homöopathie.

\section{Wissenschaft}

„Wissenschaft“ (griech. epistéme) ist zweifellos eine Erfindung der alten Griechen, im Sinne von rational begründetem Wissen. Hatte es in vorgeschichtlicher Zeit - grob gesagt - in allen Kulturen zwei Stränge der Weitergabe von Kenntnissen gegeben, die der technischen Überlieferung von praktischen Erfahrungen und Kunstfertigkeiten auf der einen und die der geistigen Überlieferung von religiösen Vorstellungen und Geboten auf der anderen Seite, so entwickelte sich in der griechischen Antike als Synthese aus den beiden Traditionen des Handwerks und des Priestertums die Philosophie [10]. Vor allem Platon und ARISTOTELES versuchten, alle praktischen und theoretischen Fragen und Probleme ihrer Zeit in ein System rationaler Begriffe, Sätze und Schlussfolgerungen zu bringen und dadurch vernunftgemäss $\mathrm{zu}$ erklären.

Das erkenntnisleitende Interesse der gesamten Antike sowie auch des Mittelalters bezog sich allerdings - im Gegensatz zur Neuzeit - auf das Was und Wozu der beobachteten Phänomene, also auf ihre ontologische und teleologische (zielgerichtete) Dimension. Bei Aristoteles, dem umfassendsten und einflussreichsten Denker und Forscher des Abendlandes, bestand Wissenschaft im Sichvergegenwärtigen und Erschliessen von sinnvollen Gebilden und Prozessen im Rahmen einer ewigen Welt- ordnung (wobei seine Begriffe und Beispiele durchwegs aus dem Handwerk und der Alltagserfahrung stammten) und kulminierte in der sogenannten theoría, einer beglückenden WesensSchau um ihrer selbst willen, die allerdings als höchste Form von práxis begriffen wurde.

So blieb dies mehr oder weniger 2000 Jahre lang, bis sich - im Rahmen und im Gefolge tiefgreifender politischer, religiöser, sozialer und ökonomischer Umbrüche (Renaissance, Reformation, Entdeckung Amerikas) - neue Erkenntnisinteressen Bahn brachen. So zielte ab dem 17. Jahrhundert Erkenntnis - im Gegensatz zur Antike fast nur noch auf die Fragen des Wie und Wodurch, also auf die funktionale und kausale Erklärung der Phänomene. Der Hintergrund der umfassenden Neubegründung von Wissenschaft durch Francis Bacon, Galileo Galilei und ISAAK NEWTON war das nunmehr erwachte und fortan dominierende Interesse am Eingreifenkönnen und Beherrschen natürlicher Prozesse und Dinge. Paradigmatisch wurde dies im 17. Jahrhundert formuliert von FRANCIS BACON in seinem Ausspruch ,Wissen ist Macht“, von René Descartes' Dictum „Wissen, um uns zu Herren und Meistern der Natur zu machen“ oder von Thomas HobBes, der im Leviathan schrieb: „Ein Ding kennen bedeutet, zu wissen, was man damit anfangen kann, wenn man es hat“. In der Folge wurde zunehmend versucht, auch Lebensvorgänge mathematisch, physikalischchemisch oder nach Prinzipien der Mechanik zu beschreiben. Einen vorläufigen Höhepunkt erreichte diese neue Form von reduktionistischer Wissenschaft im 18. Jahrhundert (1748) mit J. O. DE LA METTRIE’s Buch „L'homme machine" (der Maschinenmensch).

In seinem „Novum Organon“ hatte FrANCIS BACON zwar schon 1620 - zum Zwecke sicherer Naturbeherrschung die Beschränkung auf Erkentnisse propagiert, die durch Experiment und Erfahrung induktiv gewonnen werden. Die Wortbildung „Naturwissenschaft“ selbst findet sich allerdings erst ab dem 18. Jahrhundert, so etwa bei KANT, der 1786 zwischen „historischer“ und „rationaler“ „Naturwissenschaft“ unterschied [11].
Innerhalb der Medizin gewann das auf naturwissenschaftlicher Methodik und Tierexperimenten aufgebaute neue Forschen vor allem nach dem Ende der Naturphilosophie zunehmend an Bedeutung. So forderte Claude Bernard um 1865, lebendige Organismen ausschliesslich als physikalisch-chemisch determinierte Gebilde aufzufassen, und für Du BoIs-REYMOND bestand 1872 „naturwissenschaftliches Erkennen der Körperwelt mit Hilfe und im Sinne der theoretischen Naturwissenschaft“ nur noch in einem „Zurückführen der Veränderungen in der Körperwelt auf Bewegungen von Atomen ... oder Auflösen der Naturvorgänge in Mechanik der Atome“ [12].

Naturwissenschaftliches Denken als solches gibt es also erst seit wenigen Jahrhunderten und speziell innerhalb der Medizin - im grossen Stil - erst seit etwa 150 Jahren. Wie die wesentlich längere Kulturgeschichte der Heilkunst aber zeigt, haben Wissenschaftler und Ärzte auch schon vor der „Erfindung“ der Naturwissenschaften rational gedacht, nur eben anders. Naturwissenschaftlichkeit kann daher nur als eine bestimmte, relativ späte und spezialisierte Form von Rationalität begriffen werden - und nicht umgekehrt.

Dies gilt es $\mathrm{zu}$ beachten, wenn es darum geht, Stellung zu beziehen gegenüber leichtfertig gestellten Fragen wie „Ist die Homöopathie naturwissenschaftlich bewiesen?“ oder „Ist die Homöopathie naturwissenschaftlich widerlegt?" Wenn sich nämlich herausstellen sollte, dass Homöopathie und Naturwissenschaft, die ja beide etwa gleichzeitig innerhalb der Medizin entstanden, in entscheidenden Punkten nicht nur Gemeinsamkeiten, sondern auch prinzipielle Unterschiede aufweisen, so kann nicht erwartet werden, dass sich beider Affirmationshorizont und Begrifflichkeit einfach decken oder 1:1 ineinander übersetzbar sind. Der Wert bzw. Unwert der Prinzipien eines Heilsystems wiederum lässt sich nicht von einem anderen, anders gearteten Koordinaten-System aus feststellen. Deshalb ist eben der Einwand der Unübersetzbarkeit fraglicher Kategorien in das naturwissenschaftliche Begriffsraster noch kein prinzipielles Argument gegen sie. Naturwissenschaftliche Unbe- 
weisbarkeit ist somit nicht gleichbedeutend mit Irrationalität schlechthin.

\section{Naturwissenschaft}

Von welcher Art von Rationalität ist aber die Naturwissenschaft? Was von der Welt, vom Leben und vom Menschen erfasst sie und auf welche Weise?

Nach dem idealisierten Selbstverständnis ihrer Vertreter besteht die naturwissenschaftliche Methode in wiederholten Zyklen von Beobachten, Hypothesen bilden, Voraussagen treffen, deren experiementeller Überprüfung, Verifikation bzw. Falsifikation usw. Entscheidend und bezeichnend für die neuzeitlich-naturwissenschaftliche Art von Welt-Wahrnehmung ist jedoch die methodische Beschränkung auf die Beobachtung dessen, was exakt messbar, das heisst was quantifizierbar und reproduzierbar ist. Für die Naturwissenschaften, speziell für die Physik, existieren damit primär nur Messwerte, für deren Relationen untereinander sekundär mathematische Formeln und Gleichungen gesucht und entwickelt werden (sofern diese physikalisch-mathematische Ebene - etwa in der Quantenphysik oder Kosmologie - durch metaphysische Spekulationen verlassen wird, werden Physiker eben zu Philosophen [13]). Die Welt der Physik besteht also weder aus Menschen, Tieren oder Pflanzen, noch aus Häusern, Tischen oder Tassen, geschweige denn aus Ideen, Normen oder gar heilbaren Krankheiten, sondern ausschliesslich aus Massen, Kräften, Feldern, Wellen, (Dreh-)Impulsen, Energien, Orts- und Zeitkoordinaten usw. sowie ihren mathematischen Relationen zueinander.

Entgegen allgemeiner Überzeugung lässt sich nicht einmal der Begriff „Materie“ aus der Physik allein ableiten. So bezeichnete es der 1991 verstorbene Wissenschaftstheoretiker WoLFGANG STEGMÜLLER als „Treppenwitz des 20. Jahrhunderts“, dass der Materiebegriff der „rätselhafteste für die Wissenschaft“ sei, obwohl jeder glaube, er wisse, worum es sich dabei handelt [14]. Im Gegensatz zur Logik unserer Alltagssprache, wo sich jede Aussage über eine Eigenschaft auf ein dazugehöriges Ding beziehen muss, kommt die Physik offenbar ohne „materielles Substrat“ bzw. ohne „Träger wechselnder Eigenschaften“ aus. Für die physikalische Feldtheorie ist es zum Beispiel gleichgültig, ob man von felderzeugenden Massen spricht oder Partikel nur noch als Knoten bzw. Singularitäten im Feld ansieht. Wegen des nur relationalen Charakters physikalischer Gleichungen gibt es sowohl für die klassische Elektrodynamik als auch für die Quantentheorie logisch äquivalente Formulierungen, bei denen entweder der Partikelbegriff oder der Feldbegriff im Mittelpunkt steht.

Die Physik beschreibt also keineswegs die uns umgebende physische Welt, sondern eine stilisierte Kunstwelt.

Umso verwunderlicher ist es allerdings, dass unser modernes Bewusstsein - von unserem kosmologischen Weltbild und säkularen Menschenbild über das Bildungs- und Gesundheitswesen bis zur modernen Medizin doch vorwiegend naturwissenschaftlich geprägt ist und damit gut fundiert sein soll. Es scheint, als halten sich moderne Naturwissenschaftler erstens für zuständig für alle Bereiche unseres Daseins, zweitens für in der Lage, die Dinge unserer Lebenswelt als solche zu erfassen, und drittens für kompetent, ihr abschliessendes Urteil über sie zu fällen.

Sonderbarerweise fällt heute fast niemandem mehr auf, dass es einen Unterschied gibt zwischen dem Wesen einer Sache (bzw. einer Sache selbst) und einzelnen Messwerten davon. Die deutsche Sprache enthält diese Unterscheidung zum Beispiel durch die Begriffe „das Physische“ und „das Physikalische“, wärend die englische Sprache beides mit demselben Wort „physical“ bezeichnet. Dieser Gleichsetzung, die von einigen modernen Philosophen sogar verteidigt wird, liegt offenbar die Überzeugung zugrunde, dass das Physische, das uns lebensweltlich umgibt (also Autos, Tiere, Pflanzen usw.), genau das ist, was die Wissenschaft der Physik näher untersucht und auf den Punkt bringt. Die Wissenschaft wäre also nur eine Fortsetzung des Alltagsdenkens, und das Physische wäre das noch nicht zu sich selbst gebrachte Physikalische. Ebenso nimmt man an, Instrumente wie Mikroskope oder Fernrohre würden nur unsere gewöhnliche Weltwahrnehmung verlängern und sie verschärfen. Der Wissenschaftler betrachte also dieselbe Welt wie der Mann auf der Strasse, nur eben genauer und detaillierter.

Übersehen wird bei diesen Behauptungen allerdings, dass man zum Beispiel unter einem Rastertunnel-Mikroskop zwar Moleküle sehen kann, aber keine Tische, Steine, Wolken oder Flüsse. Gravierender noch als diese Diskontinuität in der Wahrnehmung ist aber die Diskontinuität im Beschreibungsmodus. Während wir Autos, Tiere und Pflanzen in einer natürlichen Sprache beschreiben, beschreiben wir das Ergebnis von Streuexperimenten in der hochtheoretischen, formalisierten Sprache der Mathematik, die ganz andere Eigenschaften hat als die natürliche Sprache. Zwischen der natürlichen und den formalen Sprachen gibt es aber kein Kontinuum, sondern einen deutlich wahrnehmbaren Bruch, der der Differenz zwischen physischen und physikalischen Gegenständen zugrunde liegt. Wenn ein Physiker seine Objekte mit Differentialgleichungen beschreibt, so handelt es sich dabei um mathematische Funktionen, die rein extensional definierte Mengen ineinander abbilden, das heisst um mathematische Relationen. Keines der Relate wird dabei aber als ontologisch Ursprüngliches oder als substantielle Entität ausgezeichnet, wie es in der natürlichen Sprache der Fall ist, wo ein Prädikat immer auf ein Subjekt bzw. einen Gegenstand verweist und für dessen Eigenschaft steht. Gerade aber in der Fähigkeit, etwas als etwas $\mathrm{zu}$ identifizieren, haben Philosophen von Aristoteles bis Peter F. Strawson die welterschliessende Kraft der natürlichen Sprache gesehen [15].

Wenn die Physik aber gar nicht die uns primär gegebene Lebenswelt beschreibt, sondern eine artifiziell konstruierte Kunstwelt, und darüber hinaus - durch den Erfolg der Naturwissenschaften - auch die Geisteswissenschaften unter enormen Druck geraten sind, in ihrem Bereich ebenfalls die naturwissenschaftliche Methode zu übernehmen (s. Historismus, Behaviorismus, experiementelle Psychologie, Soziobiologie, Kognitionswissenschaften usw.), so fragt sich, wo dann die uns vertraute und lebendige Welt geblieben ist, für die die Naturwissenschaft offenbar 
keine Sprache hat. Da Rationalität seit dem 19. Jahrhundert mit naturwissenschaftlicher Erklärbarkeit gleichgesetzt wird, versinken durch diesen verhängnisvollen Kurzschluss elementare Lebensdimensionen wie menschliches Handeln, Fühlen und Denken, erst recht aber Kunst, Kultur, Glaube, Liebe und Ethik oder Phänomene wie Krankheit, Gesundheit und Heilung in einer Grauzone von Irrationalität und Beliebigkeit, für die es im strengen Sinn keine wissenschaftlichen Kategorien geben soll.

Dass dieser Weltverlust aber sozusagen hausgemacht ist, das heisst, durch die geistige Reduktion aller Lebensphänomene auf quantifizierbare Messwerte selbstverschuldet, zeigt ein Blick in die Wissenschaftsgeschichte, sofern man dabei vor die sogenannte wissenschaftliche Revolution im 17. Jahrhundert zurückgeht, also zu dem vergleichsweise homogenen Zeitraum von immerhin 2000 Jahren, der mehr als von jeder anderen Philosophie vom Aristotelismus geprägt war.

\section{Aristoteles}

ARISTOTELES gilt zu Recht als Begründer der „Wissenschaft vom Lebendigen“. Im Gegensatz zu seinem Lehrer PlatoN, dessen Philosophie in einer eher statischen Ideenlehre gipfelte, ging es ARIsTOTELES darum, Bewegung (kínesis) zu erklären, und zwar im weitesten Sinne, das heisst nicht nur als Ortsbewegung, sondern auch als Werden und Vergehen sowie als quantitative und qualitative Veränderung (griech. alloíosis, metabolé). Als Grundkategorien zur wissenschaftlichen Erfassung dieser Phänomene verwendete ARISTOTELES die Begriffe Möglichkeit (griech. dýnamis, lat. potentia) und Wirklichkeit (griech. enérgeia, lat. actus). Bewegung jeder Art konnte damit allgemein begriffen werden als die Verwirklichung einer Möglichkeit, d.h. Aktualisierung einer Potenz. ArIstoteles fasst seine Theorie absichtlich so weit, dass sie - im Gegensatz zur neuzeitlichen Naturwissenschaft, die nur noch Ortsveränderungen von A nach B kennt und beobachtet - auf jede Form von Bewegung anwendbar war: auf das Wachsen einer Pflanze ebenso wie auf den Umschwung eines Gefühls oder den Wechsel der Jahreszeiten.

ARISTOTELES' Verwurzelung in der Lebenswelt und sein technisch-praktischer Zugang zur Natur zeigt sich auch in einem weiteren Grundbegriff seiner Physik, dem des „Wesens“ (griech. ousía, lat. essentia). Jedes konkret existierende Wesen lässt sich begreifen als zusammengesetzt aus seiner Materie (griech. hýle, lat. materia) und seiner Form (griech. morphé, lat. forma), wobei allerdings Materie und Form blosse Reflexionsbegriffe sind, die alleine für sich überhaupt nicht existieren können. Im Gegensatz zum neuzeitlichen Materialismus wird also gerade nicht (fälschlicherweise) behauptet, dass es so etwas wie Materie an sich einfach so geben könne, sondern alles, was wir jemals beobachten, begreifen und uns vorstellen können, ist durch die damit verbundene Formung immer schon ein Etwas, ein Wesen, und damit bereits geformte Materie. Werden (griech. génesis) stellt in diesem Sinne den Übergang von der Unbestimmtheit (Potentialität) einer primären Materie in die Bestimmtheit (Aktualität) einer Form dar, und ist letztlich so etwas wie ein Übergang ( $k i^{-}$ nesis) vom Nichts zum Sein. Klar beschreibbar ist davon aber nur das Gewordene, das das Sein als Form hat.

In der modernen Physik gibt es dagegen nur Übergänge von bestimmten $\mathrm{zu}$ anderen bestimmten Zuständen. Das Aristotelische Problem der kínesis kommt dort überhaupt nicht vor, was allein schon an der mathematischen Formulierung liegt. Mathematische Funktionen verknüpfen immer nur bestimmte Zustände miteinander. Dies ist auch der Grund, warum ARISTOTELES die Anwendung der Mathematik auf die kínesis ausschloss - wohlgemerkt nicht aus Unkenntnis der Mathematik, sondern gerade aufgrund der Einsicht in ihre Grenzen. „Von den mathematischen Gegenständen bewegt sich nämlich keiner", schrieb er in seiner Abhandlung über die Bewegung der Tiere [16]. Um das konkrete Werden zu fassen, war ARISTOTELES also gezwungen, auf die Mathematik - trotz bzw. gerade wegen der vermeintlichen „Zeitlosigkeit mathematischer Gegenstände“ zu verzichten [17].
Für die Homöopathie unmittelbar relevant könnte dieser nicht-mathematisierbare Begriff von Bewegung in dem Moment werden, als man bedenkt, dass ARISTOTELEs in analoger Weise etwa den Übergang des gesunden in den kranken Zustand eines Menschen (und umgekehrt) als qualitative Veränderung (alloíosis) betrachtete, so dass auch diese Form von Bewegung (kínesis) mit seinen Kategorien wissenschaftlichem Begreifen zugänglich war. Mit naturwissenschaftlichen Kategorien dagegen kann man nur entweder versuchen, komplexe Vorgänge wie Krankwerden oder Gesundwerden auf der Ebene von Ortsveränderungen von Molekülen zu beschreiben oder solch höhere Begriffe eben ganz zu meiden. So ist es überaus bezeichnend, dass der Begriff „Heilung“ in modernen medizinischen Wörterbüchern nicht mehr vorkommt - da er sich eben der naturwissenschaftlichen Form von Rationalität entzieht.

Von grösster Bedeutung für die Homöopathie bzw. die Medizin ganz allgemein ist eine weitere, von der neuzeitlichen Naturwissenschaft ebenfalls eliminierte Kategorie der Wissenschaft des ARISTOTELES: die Zweckgerichtetheit (Teleologie) alles Seienden. Ausgehend von der lebensweltlichen Selbst- und Welterfahrung in der griechischen Pólis sowie seinem primär technischpraktischen Verhältnis zur Natur billigte ARISTOTELES jedem Wesen ein Streben nach einem Ziel (griech. télos) zu, allerdings abgestuft: vom blinden Streben eines Steins zum Erdmittelpunkt über das bewusstlose Streben der Tiere nach Selbsterhaltung und Reproduktion der Gattung bis zum vernünftigen Streben des Menschen nach Glückseligkeit und Weisheit.

Ein bestimmter Zweck kann nun durch verschiedene Mittel erreicht werden, und auch ein bestimmtes Mittel kann verschiedenen Zwecken dienen. (Um satt zu werden, kann man zum Beispiel Wurst oder Käse essen; und mit einem Hammer kann man sowohl Nägel in die Wand als auch Fensterscheiben einschlagen). Im Gegensatz zur kausalen Verknüpfung von Ursache und Wirkung besteht zwischen Mittel und Zweck also ein Kontingenzverhältnis, was bedeutet, dass es auch andere Lösungswege geben kann. In heutiger 
Terminologie: eine many-to-many-Relation. Eindeutigkeit kann es im teleologischen Denken daher nicht geben [18].

Bei einer teleologischen Betrachtung der Natur, die primär nicht antizipierend (wie die neuzeitliche Naturwissenschaft), sondern rekonstruierend verfährt, ist zwar das Zukünftige nicht im strengen Sinne vorhersehbar (so wie sich eben bei der Saat eines Baumes dessen spätere Gestalt nicht exakt vorherbestimmen lässt), doch erlaubt andererseits der Wesens- bzw. ousíaBegriff durchaus eine Eingrenzung und Spezifizierung von Aussagen über die weitere Entwicklung einer Sache oder eines Prozesses. Da die Möglichkeiten jedes Wesens beschränkt sind, spielen sich auch deren Verwirklichungen innerhalb bestimmter Grenzen (griech. péras) ab, die man kennen kann, wenn man das Wesen erfasst hat. So wie es zum Wesen eines Hundes gehört, dass er bellen, aber nicht singen kann, so weiss auch der Ingenieur, welche Qualitäten welchem Stoff innewohnen, die ihn für bestimmte Zwecksetzungen geeignet machen.

So gesehen genügen also auch die „unverwirklichten Möglichkeiten“ einer Substanz klaren Identitätskriterien, wobei allerdings zu beachten ist, dass von einer Möglichkeit nur dann überhaupt gewusst werden kann, wenn sie schon einmal verwirklicht worden ist. (Nur der kann von sich behaupten, er könne Klavier spielen, der wirklich schon Klavier gespielt hat). Auf die Homöopathie bezogen: Dass ein bestimmtes Arzneimittel ein bestimmtes Symptom hervorrufen bzw. heilen kann, lässt sich nur dann sagen, wenn es dies schon einmal wirklich getan hat, etwa in einer Arzneimittelprüfung. Insofern kommt bei ARISTOTELES die Wirklichkeit immer vor der Möglichkeit [19].

\section{Zwei Arten Wissenschaft}

Nach diesem Exkurs in die vor-moderne Wissenschaftsgeschichte zeigt sich ein fundamentaler Unterschied zwischen zwei Prototypen von Wissenschaft.

- Auf der einen Seite die aristotelische Wissenschaft, die ihre Begriffe, Prinzipien und Konzepte aus der menschlichen Selbsterfahrung innerhalb der sinnlich erfahrbaren Lebenswelt gewinnt und nach dem Paradigma zielgerichteten Strebens und handwerklicher Herstellung von Mitteln zu bestimmten Zwecken die verschiedensten Naturphänomene und technischen Prozesse erklärt.

- Auf der anderen Seite die neuzeitliche Naturwissenschaft, die - geleitet vom säkularen Interesse an Naturbeherrschung - selektiv nur diejenigen Aspekte der Welt beobachtet und untersucht, die sich messen und wiegen sowie mathematischexakt zueinander in Relationen bringen lassen.

HAHNEMANN lebte und wirkte ziemlich genau an der Schnittstelle zwischen diesen beiden grossen Blöcken von Wissenschafts-Traditionen [20]. Wenn sich auch einige Wurzeln der neuzeitlichen Art von Naturwissenschaft bis ins 13 . Jahrhundert zu Roger BACON u.a. zurückverfolgen lassen, - zum neuen wissenschaftlichen Paradigma wurde das Experimentieren, Messen und Mathematisieren der Natur unter Gelehrten und Mäzenen erst im 17. Jahrhundert, in breiten Kreisen der Bevölkerung diskutiert wurde es erst im 18. Jahrhundert, und von der Medizin in grösserem Masse aufgegriffen erst im 19. Jahrhundert. Andererseits beherrschte der Aristotelismus noch bis weit ins 18. Jahrhundert hinein die Lehre an den Universitäten, an den medizinischen Fakultäten häufig zusammen mit Galenismus und Humoralpathologie, gegen die HaHnEMANN sein Leben lang polemisierte.

Die Zeit der Aufklärung, in die НАHNEMANN geboren wurde, war geradezu erfüllt von dem Impetus, - geblendet von den sichtbaren Erfolgen der $\mathrm{Na}$ turwissenschaften auf dem Gebiet der Technik, Landwirtschaft und Ökonomie und inspiriert vom Glauben an einen kontinuierlichen, ewigen Fortschritt - möglichst viele noch „unaufgeklärte“ Bereiche des Lebens rational, und das hiess jetzt vor allem kausalmechanisch, aufzuhellen. Analog zu Newton, der die neuzeitliche Physik als Naturwissenschaft begründet hatte, war es KANTs Anliegen gewesen, die Metaphysik zu einer streng apriori- schen Wissenschaft zu entwickeln, und so sah es HaHnEmann als seine Aufgabe, nach deren beider Vorbild endlich auch die Medizin in den Rang einer sicheren Wissenschaft zu erheben [21]. Damals, um 1800, war es - im Gegensatz zu heute - noch keineswegs ausgemacht, dass „wissenschaftliche Medizin“ eines Tages gleichbedeutend mit „naturwissenschaftlicher Medizin“ sein würde. Der ausufernde Pluralismus an Heilsystemen, der HAHNEMANN zunächst an der Medizin seiner Zeit verzweifeln liess, spiegelte vielmehr die allgemeine Umbruchsstimmung wider, die förmlich nach einem neuen einheitlichen Paradigma schrie.

\section{Homöopathie}

In diesem Sinne war es fortschrittlich von HAHNEMANN, bereits zu seiner Zeit - soweit es ging - auf die naturwissenschaftliche Methode zu setzen, die ein halbes Jahrhundert später tatsächlich durch Rudolf VIRchow, ROBERT КосH und andere der Medizin ein allgemeingültiges und heute auf der ganzen Welt anerkanntes einheitliches Paradigma bescherte (Zellularpathologie, Bakteriologie). Auch HaHnEMANN versuchte schon, seine neue Arznei-Heilkunde nach Kriterien zu begründen, die erst weit nach seinem Tod Standard geworden sind.

So führte er seine Arzneimittelprüfungen wohl deshalb an Gesunden, mit Einzelmitteln und unter strikten methodischen und diätetischen Anweisungen durch, um dem neuen Ideal des naturwissenschaftlichen Experiments nahezukommen, demzufolge an möglichst homogenen Substraten unter konstant gehaltenen Randbedingungen nur eine Variable verändert und dann das Ergebnis des Versuchs abgelesen wird. An die damals vor allem in England einflussreiche Strömung des Empirismus, die sich im nachhinein ebenfalls als zukunftsweisend für die Medizin erwies, schloss sich HAHNEMANN insofern an, als auch er - was Arzneimittelprüfungen, Anamneseerhebung und Verlaufsbeurteilungen betrifft - an die Möglichkeit reiner „vorurteilsloser“ Beobachtungen glaubte [22], eben nach dem naturwissenschaftlichen Mo- 
dell der problemlosen Registrierung objektiver Messdaten.

Dass HAHNEMANN bei seiner Reformation der Medizin das naturwissenschaftliche Ideal raum-zeit-invarianter Naturgesetzlichkeit und mithin Sicherheit und Voraussagbarkeit vorschwebte, zeigen viele Stellen in seinem Werk, etwa wenn er behauptete, die Homöopathie werde einst „den mathematischen Wissenschaften an Zuverlässigkeit nahe kommen“ [23]. Was dazu noch fehle, seien lediglich genaue „Beobachtungen“ mehrerer Arzneimittelprüfer - so räsonierte er weiter -, was wiederum ein bezeichnendes Licht auf Hahnemanns Anlehnung an die damals ebenfalls progressive naturwissenschaftliche Methode der Induktion wirft, nach der aus möglichst vielen Einzelbeobachungen allgemeine Gesetzmässigkeiten abgeleitet werden sollen.

Soweit einige naturwissenschaftliche Anteile an HAHNEMANNs Entwurf einer rationalen Arznei-Heilkunde. Damit allein liess und lässt sich aber noch keine Homöopathie begründen. (Wäre das möglich, wäre sie längst an den Universitäten anerkannt bzw. zur Schulmedizin geworden).

Zusammengehalten wurde das Heilsystem HaHnEMANNs als Ganzes vielmehr durch die Klammer des Rationalismus, einer mächtigen Denkströmung des 18. Jahrhunderts, die von der Voraussetzung ausging, dass der Welt Vernunft zu Grunde liege - die wiederum der Mensch mit seiner Vernunft erkennen könne. Dieser Vernunftbegriff war nicht auf die Kategorien der Naturwissenschaft eingeengt, so dass praktisch alle Bereiche des Lebens thematisiert werden konnten, wie Natur, Kultur, Religion, Anthropologie, Ethik usw. Indem HAHNEMANN sozusagen mit einem Bein noch in dieser Tradition stand, die wiederum so etwas wie ein neuzeitlicher Ausläufer des Aristotelismus war, konnte er gleichzeitig Begriffe und Argumentationsmuster benutzen, die mit dem zunehmend in die Medizin einsickernden naturwissenschaftlichen Ansatz inkompatibel waren [24].

Bei Begriffen wie Krankheits- oder Arznei-„Potenzen“ hört man förmlich die aristotelische Kategorie der „Möglichkeit“ heraus (lat. potentia), während hinter Begriffen wie „Dynamis“ oder „dynamisch“ das gleiche Wort steckt, nur eben in griechischer Sprache (griech. dýnamis). HAHNEMANns Begriff der „Lebenskraft“ wiederum wirkt wie der Versuch einer rationalistischen Fassung von ARIstoteles' Konzept der „Entelechie“ (griech. entelecheía: das zielgerichtete Streben von Lebewesen), die im Gefolge von NEwTons Physik allerdings in naturwissenschaftlicher Terminologie, daher eben als Kraft, ausgedrückt werden musste. Auch das Simile-Prinzip steht letztlich quer zum naturwissenschaftlichen Begriffsrahmen, ist allerdings anschlussfähig an das aristotelisch-scholastische Analogiedenken bzw. den antiken Analogieschluss. Um der Begründbarkeit des Ähnlichkeits-Prinzips als einzig möglichem und wahrem Heilprinzip willen war HAHNEMANN letztlich gezwungen, auf Glaubenssätze des Rationalismus wie die Güte und Weisheit des Schöpfers und die hohe geistige und sittliche Bestimmung des Menschen zurückzugreifen [25], was alles wiederum auf der aristotelischen Teleologie-Lehre beruhte.

Wie sich nach diesen Beispielen erkennen lässt, hat die Homöoapthie mindestens zwei Wurzeln, die sich historisch auf verschiedene WissenschaftsTraditionen zurückführen lassen:

- Einerseits standen dem Praktiker HAHNEMANN während der Umbruchszeit um 1800 noch die primär lebensweltlich-praktischen Kategorien der griechischen, lateinischen und arabischen Klassiker (kurz des Aristotelismus) zur Verfügung,

- andererseits war der Theoretiker HAHNEMANN bereits erfasst von dem Drang, die Medizin zu einer Naturwissenschaft im Sinne voraussagbaren, mathematisch-sicheren Wissens zu machen.

Insofern finden sich in der Homöopathie sowohl progressiv-naturwissenschaftliche als auch traditionell-teleologische Elemente in einer nur schwer entwirrbaren Mischung, was sie anfällig macht für alle Arten von Interpretationen und „Weiterentwicklungen“. Dies ist der Hintergrund für die aktuelle, wohl kaum in absehbarer Zeit aufzulösende Homöopathie-Debatte.

\section{Die wissenschafts- historische Sicht}

Aus wissenschaftshistorischer Sicht zu bedenken ist vor allem dreierlei:

1. Zunächst ist festzustellen, dass die Homöopathie eine praktische Tätigkeit (griech. práxis) zur Heilung von kranken Menschen ist, deren Erfolge bei individuellen Kranken und deren Verbreitung und Popularität rund um die Welt [26] für sich selbst sprechen. Indem sie nach einer Methode vorgeht, die nach traditionell-wissenschaftlichen Kriterien strukturiert und nachvollziehbar ist, ist sie eine praktische Wissenschaft - zumindest im klassisch-aristotelischen Sinne. Dies erkennen und anerkennen zu können, ist in der heutigen Zeit nicht immer leicht, da man zu sehr auf $\mathrm{Na}$ turwissenschaftlichkeit fixiert ist, doch wäre dies ein solider Standpunkt. Abzuraten ist dagegen von der Versuchung, mehr über die Homöopathie zu behaupten, als sie von ihren Prinzipien hergibt („Heilungsgewissheit“ o.ä.). Umgekehrt kann und braucht - entsprechend НАНNEMANns Position von 1796 [27] - nicht ausgeschlossen zu werden, dass naturwissenschaftliche Grundlagenforschung oder klinische Studien künftig noch manches Licht auf Teilaspekte der Homöopathie werfen könnten. Als Ganzes wird die Homöopathie allerdings - ebensowenig wie die Schulmedizin - nie in moderne Wissenschaft aufgehen können, da letztere immer nur auf die Gewinnung von allgemeinen Erkenntnissen abzielt, ärztliche Praxis dagegen auf die Behandlung von individuellen Menschen $[28,29]$.

2. Der Anspruch, dass die Homöopathie Naturwissenschaft im neuzeitlichen Sinne sei, mag aus der Sicht HAHNEMANNS verständlich gewesen sein. In der allgemeinen Fortschritts-Euphorie seiner Zeit konnte man sich noch nicht vorstellen, dass die Anwendung von Naturwissenschaft der Menschheit nicht nur Nutzen, sondern auch Gefahren und Katastrophen bringen kann. Insofern mag heute diese Etikettierung nicht mehr so attraktiv erscheinen wie damals.

Was zu Beginn der Moderne an naturwissenschaftlichen Prinzipien als progressiv und zukunftsversprechend erschien, ist inzwischen, aus postmoderner Perspektive, ausserdem längst 
unter die Räder des Dekonstruktivismus gekommen. Der Empirismus zum Beispiel wurde vom Konstruktivismus als naive Illusion demaskiert, mit dem Argument, jede Beobachtung sei wesentlich mehr Konstruktion von seiten des Subjekts als neutrale Perzeption von Objekten [30]. Die naturwissenschaftliche Methode der Induktion und Falsifikation à la POPPER wurde von Wissenschaftstheoretikern wie THомаS Kuhn [31] oder PAul Feyerabend [32] als selbstgefällige Ideologie entlarvt, zumal der reale Wissenschafts-Betrieb eher sozialen und monetären Interessen folge als den vorgeblichen Kriterien zur Wahrheitsfindung. Das Konzept von linearer Kausalität, Berechenbarkeit und Vorhersagbarkeit der Welt, auf dem die Newtonsche Physik beruhte, wurde schliesslich von der Chaosforschung dahingehend relativiert, dass es sich dabei höchstens um einen Spezialfall (in einem künstlich-geschlossenen System) innerhalb eines Universums von nichtlinearen Prozessen handelt [33].

Es sieht so aus, als wäre Naturwissenschaftlichkeit, was ihre Fundierung und Folgekosten anbelangt, heute selbst in die Krise und Kritik geraten, so dass sie für Mediziner möglicherweise nicht mehr die beste - geschweige denn ausschliessliche - Verbündete zu sein scheint.

3. Vor dem Hintergrund ökologischer Katastrophen und alarmierender $\mathrm{Ne}$ benwirkungen schulmedizinischer Medikamente werden die Langzeitschäden einer ungezügelten Herrschaft der Naturwissenschaft über alle Lebensbereiche heute immer kritischer gesehen. So stellt sich nicht nur für die Homöopathie, sondern auch für die Gesellschaft als Ganzes die Frage nach einem besser ausbalancierten Verhältnis zwischen naturwissenschaftlicher Theorie und lebensweltlicher Praxis. Wenn die Prädominanz naturwissenschaftlicher Theorien in Bezug auf unser modernes Weltbild und unser Handeln immer deutlicher mit der Gefahr eines physischen, psychischen und geistigen Weltverlustes einhergeht, so erfordert diese naturwissenschaftliche Theorielastigkeit unseres Weltbezugs offensichtlich ein Gegengewicht durch komplementäre Weltzugänge, die die lebensweltliche Praxis höher gewichten.
Was zum Beispiel ein Mensch, was ein Lehrer oder was ein Homöopath ist, weiss man am besten und unmittelbarsten dann, wenn man selbst einer ist, das heisst, wenn man vom eigenen praktischen Vollzug her sein Wesen durchschaut, damit umgehen kann und es - nach Möglichkeit - in wissenschaftliche Begriffe fassen kann. Da im Gegensatz dazu - die theoretische Physik von praktischen und lebendigen Dingen nichts versteht, ja nicht einmal sagen kann, was Materie ist, wird von ihr und der an ihr orientierten Naturwissenschaft auch keine Aufklärung des Wesens der Homöopathie oder Ähnliches zu erwarten sein.

Hat man einmal verstanden, dass Wissenschaft-Treiben selbst bereits eine menschliche Tätigkeit ist, die den Menschen (den sie erklären soll) und seine Praxis immer schon voraussetzt, so ist der erste Schritt zu einer Neubestimmung des Stellenwerts naturwissenschaftlicher Theorie innerhalb unseres Lebens sowie unserer Medizin getan. In diesem Zusammenhang könnten lebensweltlich-praktische Kategorien, wie sie in Aristoteles’ „Wissenschaft vom Lebendigen“ vorliegen, künftig zu neuer, ungeahnter Bedeutung gelangen. Wie im Vorhergehenden zu zeigen versucht wurde, könnte die Homöopathie davon nur profitieren.

\section{Literatur}

1. Hahnemann S: Versuch über ein neues Prinzip zur Auffindung der Heilkräfte der Arzneisubstanzen (1796). In: Schmidt JM, Kaiser D (Hrsg): Samuel Hahnemanns Gesammelte kleine SchrifSamuel Hahnemanns Gesammelte kleine

2. Hahnemann S: Heilkunde der Erfahrung (1805). In: Schmidt JM, Kaiser D (Hrsg.): Samuel Hahnemanns Gesammelte kleine Schriften. Heidelberg, Haug, 2001, 387-417.

3. Hahnemann S: Fingerzeige auf den homöopathischen Gebrauch der Arzneien in der bisherigen Praxis (1807). In: Schmidt JM, Kaiser D (Hrsg): Samuel Hahnemanns Gesammelte kleine Schriften. Heidelberg, Haug, 2001, 460-472, hier 461.

4. Schmidt JM: Die Entstehung, Verbreitung und Entwicklung von Heilsystemen als Gegenstand der Medizingeschichte - am Beispiel der Homöopathie. Sudhoffs Archiv 2007:91:38-72.

5. Habich K, Kösters C, Rohwer J (Hrsg): Sinnvoller Fortschritt oder Rückschritt in die Zeit vor Hahnemann? Eine internationale Diskussion um moderne Strömungen, Methodenvielfalt und genuine Homöopathie, mit Beiträgen, Kommentaren und Texten von J. Winston, A. Saine, [usw.] und ca. 70 weiteren Autoren (http://www.grundlagenpraxis.de, News, Grundlagendebatte)

6. Wichmann J: Die andere Wirklichkeit der Homöopathie. Eine Heilweise zwischen Alchemie, Schamanismus und Wissenschaft. Saarbrücken, Neue Welt, 2002.

7. Fräntzki E: Die Idee der Wissenschaft bei Samuel Hahnemann. Heidelberg, Haug, 1976.

8. Schüppel R: Evidenzbasierte Homöopathie (EBH). Irrglaube aus der Schulmedizin oder Gebot der Stunde? Allg hom Ztg 2003:248:173-184.
9. Gypser KH: Homöopathie. Grundlagen und Praxis. München, C.H. Beck, 1998.

10. Mason SF: Geschichte der Naturwissenschaft in der Entwicklung ihrer Denkweisen. Stuttgart, Verlag für Geschichte der Naturwissenschaften und Technik, 1991

11. Kant I: Metaphysische Anfangsgründe der Naturwissenschaft. Riga, 1786, A IV-VI.

12. Schmidt JM: Homöopathie und Philosophie. Versuch einer philosophischen Rekonstruktion de Begründung der Homöopathie durch Samue Hahnemann. Scheidewege 1990/91;20:141-165.

13. Scheibe E: Die Philosophie der Physiker. München, C.H. Beck, 2007.

14. Stegmüller W: Hauptströmungen der Gegenwartsphilosophie. Bd. 2. Stuttgart, Kröner, 1989, 585.

15. Mutschler HD: Naturphilosophie. Stuttgart, Kohhammer, 2002, 90-96.

16. Aristoteles: De motu animalium, 698 a27

17. Leiber T: Kosmos, Kausalität und Chaos. Naturphilosophische, erkenntnistheoretische und wissenschaftliche Perspektiven. Würzburg, Ergon, 1996, 101-104.

18. Mutschler HD: Naturphilosophie. Stuttgart, Kohlhammer, 2002, 133-151.

19. Spaemann R, Löw R: Die Frage Wozu? Geschichte und Wiederentdeckung des teleologischen Denkens. München, Piper, 1985, 51-78; hier 57.

20. Schmidt JM: Taschenatlas Homöopathie in Wort und Bild. Grundlagen, Methodik und Geschichte. Heidelberg, Haug, 2001

21. Schmidt JM: Grundlagen und Entwicklungen in der Homöopathie. Dt med Wschr 1993;118: 1085-1090.

22. Wischner M: Organon-Kommentar. Eine Einführung in Samuel Hahnemanns Organon der Heilkunst. Essen, KVC Verlag, 2001, 18-23.

23. Hahnemann S: Organon der Heilkunst (1842) Neufassung der 6. Auflage mit Systematik und Glossar, hrsg. von Schmidt JM. 2. Aufl. München, Elsevier, 2006, § 145/1.

24. Schmidt JM: Die philosophischen Vorstellungen Samuel Hahnemanns bei der Begründung der Homöopathie. München, Sonntag, 1990.

25. Schmidt JM: Believing in order to understand Hahnemann's hierarchisation of values. Homeopathy 2008;97:156-160.

26. Dinges M (Hrsg.): Weltgeschichte der Homöopathie. Länder, Schulen, Heilkundige. München C.H. Beck, 1996

27. Schmidt JM: Der Simile-Weg als "deuteros plous" in der Arzneitherapie - Konzeption und Rezeption. Documenta Homoeopathica 1992:12: $51-59$

28. Wieland W: Diagnose. Überlegungen zur Medizintheorie. Warendorf, Verlag Johannes G. Hoof 2004.

29: Wiesing U: Wer heilt, hat Recht? Über Pragmatik und Pluralität in der Medizin. Stuttgart, Schattauer, 2004

30. Foerster $\mathrm{H}$ von, Glasersfeld $\mathrm{E}$ von: Wie wir uns erfinden. Eine Autobiographie des radikalen Konstruktivismus. Heidelberg, Carl-Auer-SystemeVerlag, 1999

31. Kuhn TS: Die Struktur wissenschaftlicher Revolutionen. 2. Aufl. Frankfurt, Suhrkamp Taschenbuch Verlag, 1976.

32. Feyerabend $\mathrm{P}$. Wider den Methodenzwang Frankfurt, Suhrkamp Taschenbuch Verlag, 1986

33. Küppers G: Chaos und Ordnung. Formen de Selbstorganisation in Natur und Gesellschaft. Stuttgart, Reclam, 1996

\section{Disclosure Statement}

The author declares that no financial or other conflict of interests exists in relation to the content of this article.

\section{Korrespondenzadresse}

PD Dr. med. Dr. phil. Josef M. Schmidt Institut für Geschichte der Medizin Ludwig-Maximilians-Universität München Lessingstr. 2, DE-80336 München

j.m.schmidt@Irz.uni-muenchen.de 